:

ISSN: 2178-7514

Vol. 12 | No. 3 | Ano 2020
ARTIGO DE REVISÃO

\section{ALTERAÇÕES DE LINGUAGEM ORAL NA APRAXIA DE FALA IDIOPÁTICA INFANTO-JUVENIL: UMA REVISÃO INTEGRATIVA}

Oral language changes in idiopathic speech apraxia in children and adolescents: an integrative review
Yasmin Valente Machado ${ }^{1}$; Jadenn Rubia Lima Costa ${ }^{2}$; Julianna Lacerda Facundo ${ }^{3}$ Myllena Gomes da Silva ${ }^{4}$

\title{
RESUMO
}

A apraxia de fala é uma desorganização motora, prejudicando a programação e execução dos movimentos orofaciais fundamentais para a realização dos fonemas, e quando atinge as crianças, elas sentem mais dificuldades se comparadas a crianças que possuam distúrbios de fala e linguagem de outros gêneros. O objetivo do estudo foi verificar o cenário nacional e internacional através de publicações atuais sobre as principais alterações identificadas na linguagem oral de pacientes infantis e jovens apráxicos idiopáticos. Trata-se de uma revisão integrativa de literatura, por meio de buscas nas bases de dados Scielo, Lilacs, Pubmed, PMC (Pubmed Central), BVS (Biblioteca Virtual em Saúde) e Periódicos Capes, contemplando o período entre 2009 a 2019, na qual foram utilizados os descritores "Apraxias", "Fala", "Fonoaudiologia", além do termo "Infância", em português e inglês. Foram encontrados através dos descritores utilizados o total de 326 artigos, porém após filtrá-los inicialmente com o descarte pelo título e resumo, e logo após com os critérios de inclusão e exclusão, restaram apenas 34, porém como havia repetição de artigos, foram selecionados 16, excluindo-se dezoito artigos. A pesquisa revelou poucos estudos, o que revela a necessidade de mais pesquisas não-generalistas, ou seja, que busquem mais dados científicos voltados para as diferentes origens do distúrbio e públicos também diversos.

Palavras-chave: Apraxias. Fala. Fonoaudiologia. Infância.

\section{ABSTRACT}

Apraxia of speech is a motor disorganization, harming the programming and execution of orofacial movements fundamental for the realization of phonemes, and when it reaches children, they feel more difficulties if compared to children who have speech and language disturb of other genres. The aim of the study was to verify the national and international scenario through current publications on the main changes identified in the oral language of pacients childish and young with idiopathic apraxia. It is an integrative literature review, through searches in the databases Scielo, Lilacs, Pubmed, PMC (Pubmed Central), VHL (Virtual Health Library) and Capes Periodicals, covering the period from 2009 to 2019, in which used the descriptors "Apraxias", "Speech", "Speech Therapy", in addition to the term "Childhood", in Portuguese and English. A total of 326 articles were found through the descriptors used, but after initially filtering them by discarding them by title and abstract, and just after with the inclusion and exclusion criteria, only 34 remained, however as there was repetition of articles, they were selected 16, being excluded eighteen articles. The research revealed few studies, which reveals the need for more research not generalist, that is, to seek more scientific data aimed at the different origins of the disturb and also miscellaneous audiences.

Keywords: Apraxias. Speech. Speech Therapy. Childhood.

\footnotetext{
1 - Especialista em Saúde do Adulto e do Idoso (Atenção em Clínicas Médica e Cirúrgica, Residência Multiprofissional HU-UFMA). Pós Graduada em Distúrbio da Fala e da Linguagem (FAVENI). Pós Graduanda em Fonoaudiologia no Transtorno do Espectro Autista (Centro Universitário Celso Lisboa).

2 - Pós-graduada em Fonoaudiologia Hospitalar com Enfoque em Disfagia (ESAMAZ). Mestranda em Meio Ambiente (Universidade Ceuma). Pós-graduanda em Distúrbio da Fala e da Linguagem (Faculdade Futura). Preceptora da Universidade Ceuma no curso de Fonoaudiologia.

3 - Graduada em Fonoaudiologia (Universidade Ceuma).

4 - Graduada em Fonoaudiologia (Universidade Ceuma).
}

Autor de correspondência

Yasmin Valente Machado

yasminvalente54@hotmail.com 


\section{INTRODUÇÃO}

A apraxia de fala é uma desorganização motora que prejudica a programação e execução de uma sequência de movimentos orofaciais fundamentais para a realização dos fonemas, e pode atingir quaisquer faixas etárias, segundo Silva ${ }^{(1)}$.

A American Speech-Language-Hearing Association $^{(2)}$ mostra que as apraxias podem ser ocasionadas por algumas circunstâncias, como associação com patologias neurológicas determinadas, ou transtornos neurocomportamentais complexos, mas também ser de origem idiopática (a alteração da fala pode estar ligada à uma falha neurogênica idiopática).

Em seu estudo, Shriberg et al..$^{(3)}$ estabeleceram sinais significativos que contribuem para um diagnóstico de apraxia, como tateio articulatório, grande quantidade de erros em vogais, substituições, inconsistência de trocas e também no uso do acento.

Direcionado para o público infantojuvenil, população esta que se encontra em fase de desenvolvimento, Lewis et al.(4) realizaram comparações entre conjuntos de crianças apráxicas de origem idiopática com conjuntos de crianças que possuíam distúrbios de fala e linguagem de outros gêneros, e descobriram que quando ambos eram expostos ao intenso volume de informações recebidas no período escolar, as crianças apráxicas encontravam mais dificuldades de adaptação.

Souza e Payão(5) esclareceram que apesar das crianças com apraxias idiopáticas apresentarem comprometimentos parecidos aos manifestados em adultos apráxicos de forma adquirida, ambos os casos exibem características específicas, o que as tornam clinicamente diferentes.

Conforme Iuzzini e Forrest ${ }^{(6)}$ defenderam em sua pesquisa, a apraxia é um dos distúrbios cujas características permanecem por mais tempo em um indivíduo e que possuem um método terapêutico mais difícil, se comparada com outros distúrbios de linguagem, além do fato de que é frequentemente confundida também com este grupo, apesar de possuir sinais singulares, o que explica a importância da criação de protocolos avaliativos capazes de proporcionar uma diminuição nos erros de diagnóstico e uma atuação precoce profissional sobre o distúrbio.

A partir das questões voltadas sobre quais seriam as alterações de linguagem oral manifestadas em indivíduos apráxicos idiopáticos que poderiam se classificar como critérios de diagnóstico, o objetivo do trabalho foi verificar o cenário nacional e internacional através de publicações atuais sobre as principais alterações identificadas na linguagem oral de pacientes infantis e jovens apráxicos idiopáticos. 


\section{MÉTODO}

Trata-se de uma revisão bibliográfica integrativa da literatura sobre a linguagem oral de pacientes infantis e jovens com apraxia de fala idiopática. O questionamento para a pesquisa foi: Qual a construção existente de conhecimentos sobre as alterações de linguagem oral manifestadas por pacientes apráxicos infanto-juvenis idiopáticos?

O levantamento dos artigos científicos foi realizado através das bases de dados Scielo, Lilacs, PubMed, PubMed Central(PMC), BVS (Biblioteca Virtual em Saúde) e Periódicos Capes. Na busca dos artigos foram utilizados os descritores em português e inglês e suas combinações "Apraxias", "Fala", "Fonoaudiologia", além do termo "Infância", devido a dificuldade de encontrar artigos relacionados à faixa etária alvo da pesquisa.

Os artigos encontrados na busca foram analisados pelas pesquisadoras responsáveis, e quanto aos critérios de inclusão foram aceitos artigos originais publicados nos anos de 2009 a 2019 nos idiomas inglês, português ou espanhol, e que abordavam temas relacionados à linguagem oral de sujeitos com apraxia de fala de origem idiopática, nas populações infantis e jovens (menores de 18 anos), com diagnóstico e registro de alterações. Os critérios definidos para a exclusão das pesquisas foram artigos em que as alterações de linguagem oral manifestadas por indivíduos diagnosticados não ficaram devidamente descritas, e também artigos em que os pacientes apráxicos possuíam quaisquer outras desordens de origens neurológicas, neurogenéticas, adquiridas, etc.

A organização dos dados foi realizada a partir da elaboração de uma estrutura contendo os nomes dos autores e ano da publicação, base de dados; país no qual a pesquisa foi realizada; e o delineamento do estudo. A análise dos artigos selecionados ocorreu por meio da leitura e crítica dos títulos, resumos e dos conteúdos completos das pesquisas onde se buscaram as alterações identificadas na linguagem oral dos sujeitos com apraxia de fala. Logo após, as alterações semelhantes foram agrupadas e contabilizadas no que tange ao aparecimento nos artigos.

\section{RESULTADOS}

Foram encontrados através dos descritores utilizados o total de 326 artigos, porém após filtrá-los inicialmente com o descarte pelo título e resumo, e logo após com os critérios de inclusão e exclusão, restaram apenas 34, porém como havia repetição de artigos, foram selecionados 16, excluindo-se dezoito artigos (Quadro 1). 


\begin{tabular}{|c|c|c|c|c|}
\hline $\begin{array}{l}\text { Bases de Dados e } \\
\text { Combinação de } \\
\text { Descritores }\end{array}$ & $\begin{array}{c}\text { Artigos } \\
\text { encontrados }\end{array}$ & $\begin{array}{c}\text { Artigos } \\
\text { descartados } \\
\text { pelo titulo e } \\
\text { resumo } \\
\end{array}$ & $\begin{array}{c}\text { Artigos que não } \\
\text { se encaixam na } \\
\text { pesquisa }\end{array}$ & $\begin{array}{c}\text { Artigos } \\
\text { incluidos }\end{array}$ \\
\hline \multicolumn{5}{|l|}{ Scielo } \\
\hline $\begin{array}{c}\text { Apraxias AND fala OR } \\
\text { fonoaudiologia }\end{array}$ & 19 & 14 & - & 5 \\
\hline $\begin{array}{l}\text { Apraxias AND speech } \\
\text { AND childhood }\end{array}$ & 3 & - & 2 & 1 \\
\hline \multicolumn{5}{|l|}{ Lilacs } \\
\hline Apraxias AND fala & 30 & 23 & 4 & 3 \\
\hline $\begin{array}{c}\text { Apraxias } \\
\text { AND fonoaudiologia }\end{array}$ & 5 & 4 & 1 & - \\
\hline $\begin{array}{l}\text { Apraxias AND speech } \\
\text { AND childhood }\end{array}$ & 7 & 5 & 1 & 1 \\
\hline \multicolumn{5}{|l|}{ PubMed } \\
\hline $\begin{array}{l}\text { Apraxias AND } \\
\text { fonoaudiologia }\end{array}$ & 3 & 1 & 1 & 1 \\
\hline Apraxias AND fala & 2 & - & - & 2 \\
\hline \multicolumn{5}{|l|}{ PubMed Central (PMC) } \\
\hline $\begin{array}{l}\text { Apraxias AND speech } \\
\text { AND childhood }\end{array}$ & 81 & 65 & 13 & 3 \\
\hline \multicolumn{5}{|l|}{ Periódicos CAPES } \\
\hline Apraxias AND fala & 24 & 15 & 4 & 5 \\
\hline $\begin{array}{l}\text { Apraxias AND } \\
\text { fonoaudiologia }\end{array}$ & 10 & 6 & 2 & 2 \\
\hline \multicolumn{5}{|l|}{$B V S$} \\
\hline $\begin{array}{l}\text { Apraxias AND speech } \\
\text { AND childhood }\end{array}$ & 142 & 91 & 40 & 11 \\
\hline
\end{tabular}

Fonte: Elaborado pelas autoras (2020).

A maioria dos artigos foi encontrada na base de dados BVS, com 7 artigos (43,75\%), seguida da Scielo com 5 artigos (31,25\%), PubMed Central com 3 artigos (18,75\%), Periódicos CAPES com 1 artigo (6,25\%), e no Lilacs não houve artigos incluídos após eliminação de pesquisas repetidas. Do total de 16 artigos, 6 foram realizados no Brasil (37,5\%), 6 estudos foram realizados nos Estados Unidos
(37,5\%), 1 na Austrália (6,25\%), 1 no Irã (6,25\%), 1 na África do Sul (6,25\%) e 1 na Suécia (6,25\%). Quanto ao delineamento do estudo, 7 eram estudos analíticos e transversais (43,75\%), 3 experimentais $(18,75 \%), 2$ estudos analíticos e longitudinais (12,5\%), 2 descritivos (12,5\%), 1 estudo de caso-controle (6,25\%) e 1 estudo de coorte $(6,25 \%)$. Os artigos selecionados dataram entre os anos de 2009 a 2019 (Quadro 2).

Quadro 2. Artigos selecionados para a revisão.

\begin{tabular}{|c|c|c|c|}
\hline Autores/Ano do estudo & Base de Dados & Pais & Delineamento \\
\hline Rechia et al. ${ }^{(7)}-2010$ & Scielo & $\mathrm{BR}$ & $\begin{array}{c}\text { Estudo analítico, transversal, } \\
\text { observacional }\end{array}$ \\
\hline Rechia et al. ${ }^{(8)}-2009$ & Scielo & $\mathrm{BR}$ & $\begin{array}{c}\text { Estudo analítico, transversal, } \\
\text { observacional }\end{array}$ \\
\hline Almeida-Verdu et al. ${ }^{(9)}-2015$ & Scielo & BR & Estudo experimental, prospectivo \\
\hline Rechia e Souza ${ }^{(10)}-2010$ & Scielo & BR & Estudo descritivo, qualitativo \\
\hline $\begin{array}{l}\text { Catrini e Lier-DeVitto } \\
2019\end{array}$ & Scielo & BR & Estudo descritivo, relato de caso \\
\hline Grigos et al. ${ }^{(12)}-2015$ & PMC & USA & Estudo experimental, prospectivo \\
\hline Grigos e Kolenda $^{(13)}-2010$ & PMC & USA & Estudo de caso-controle, analítico \\
\hline Fedorenko et al. ${ }^{(14)}-2016$ & PMC & USA & $\begin{array}{c}\text { Estudo de coorte, analítico, } \\
\text { observacional }\end{array}$ \\
\hline Keske-Soares et al. ${ }^{(15)}-2018$ & CAPES & BR & Estudo transversal, quantitativo \\
\hline Murray et al. ${ }^{(16)}-2019$ & BVS & AUS & $\begin{array}{c}\text { Estudo analítico, transversal, } \\
\text { retrospectivo }\end{array}$ \\
\hline Shakibayi et al. ${ }^{(17)}-2019$ & BVS & IRI & $\begin{array}{c}\text { Estudo analítico, transversal, } \\
\text { observacional }\end{array}$ \\
\hline Merwe e Steyn ${ }^{(18)}-2018$ & BVS & RSA & Estudo experimental, prospectivo \\
\hline Malmenholt et al. ${ }^{(19)}-2017$ & BVS & SWE & $\begin{array}{l}\text { Estudo analítico, transversal, } \\
\text { observacional }\end{array}$ \\
\hline Grigos e Case $^{(20)}-2016$ & BVS & USA & Estudo analítico, longitudinal \\
\hline Overby e Caspari(21) - 2015 & BVS & USA & Estudo analítico, longitudinal \\
\hline Preston et al. ${ }^{(22)}-2014$ & BVS & USA & $\begin{array}{c}\text { Estudo analítico, transversal, } \\
\text { observacional }\end{array}$ \\
\hline
\end{tabular}

Fonte: Elaborado pelas autoras (2020). 
No Quadro 3, estão descritas as alterações apráxicos estudados pelos autores dos artigos de linguagem oral encontradas nos indivíduos selecionados.

Quadro 3. Descrições das alterações de linguagem oral encontradas nos artigos selecionados.

\begin{tabular}{|c|c|}
\hline Autores & Alterações de linguagem oral \\
\hline Rechia et al. ${ }^{(7)}$ & $\begin{array}{l}\text { Exclusões em: } \\
\text { - sílabas, principalmente nesta frequência: sílaba pré-tônica, a pré-pré-tônica, a pós-pós- } \\
\text { tônica e, a pós-tônica } \\
\text { - sílabas, em nasais e plosivas } \\
\text { - consoantes em sílabas pós-pós-tônicas } \\
\text { - consoantes em líquidas } \\
\text { - vogais em sílabas pré-pré-tônica e pré-tônica } \\
\text { Poucas formações de palavras polissilábicas e trissilábicas }\end{array}$ \\
\hline Rechia et al. ${ }^{(8)}$ & $\begin{array}{l}\text { Assimilações em palavras trissilábicas e polissilábicas } \\
\text { Variaços articulatórias em silaba tônica } \\
\text { Substituições usuais em líquidas e fricativas, além de substituições idiossincráticas em } \\
\text { palavras trissilábicas }\end{array}$ \\
\hline Almeida-Verdu et al. ${ }^{\text {(श) }}$ & $\begin{array}{l}\text { Inconsistência nos erros } \\
\text { Dificuldade em combinar sons } \\
\text { Pausas aumentadas entre palavras, sílabas e sons } \\
\text { Simplificação de palavras } \\
\text { Exclusão de sílabas e sons } \\
\text { Articulação inadequada }\end{array}$ \\
\hline Rechia e Souza $^{(10)}$ & $\begin{array}{l}\text { Limitação extrema de silabas } \\
\text { Restrição de consoantes } \\
\text { Variacões articulatórias }\end{array}$ \\
\hline Catrini e Lier-DeVitto ${ }^{\mid 11)}$ & $\begin{array}{l}\text { Variação de articulações consolidadas, resistentes à mudança } \\
\text { Reiteração de oposiçôes consonantais com sons parecidos, associados também com } \\
\text { vogais } \\
\text { Inconsistência de fonemas } \\
\text { Alteração da pauta acentual e da entonação }\end{array}$ \\
\hline Grigos et al. $^{(12)}$ & $\begin{array}{l}\text { Inconsistência de erros em consoantes e vogais } \\
\text { Problemas em formar transiçôes articulatórias } \\
\text { Distorções } \\
\text { Metáteses } \\
\text { Aumento na proporção de erros a partir de expressões mais extensas e complexas }\end{array}$ \\
\hline Grigos e Kolenda $^{(13)}$ & $\begin{array}{l}\text { Inconsistência de erros em consoantes e vogais (omissões, substituições, distorções) } \\
\text { Metáteses }\end{array}$ \\
\hline 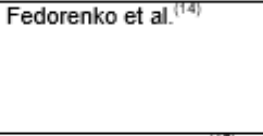 & $\begin{array}{l}\text { Fonemas imprecisos } \\
\text { Exclusão de consoante em posição final } \\
\text { Exclusão de sílaba átona } \\
\text { Simplificação de palavras } \\
\text { Aumento na proporção de erros a partir de expressões mais extensas e complexas }\end{array}$ \\
\hline Keske-Soares et al. ${ }^{(15)}$ & $\begin{array}{l}\text { Dificuldades na estrutura consoante-vogal-consoante em monossílabas } \\
\text { Dissílabas com sîlabas duplicadas, com mesma consoante e, principalmente, palavras } \\
\text { polissilábicas }\end{array}$ \\
\hline Murray et al. ${ }^{(1 t)}$ & Inconsistência nos erros \\
\hline Shakibayi et al. ${ }^{[17)}$ & $\begin{array}{l}\text { Inconsistência nos erros } \\
\text { Mau sequenciamento de consoantes } \\
\text { Articulação inadequada } \\
\text { Dificuldade com palavras multissilábicas } \\
\text { Metáteses }\end{array}$ \\
\hline Merwe e Steyn ${ }^{(t) t)}$ & Distorcōes \\
\hline Malmenholt et al ${ }^{(19)]}$ & $\begin{array}{l}\text { Inconsistência nos erros em consoantes e vogais } \\
\text { Mau sequenciamento }\end{array}$ \\
\hline Griqos e Case $^{(20)}$ & Inconsistência das consoantes \\
\hline Overby e Caspari ${ }^{|2| \mid}$ & $\begin{array}{l}\text { Dificuldades na estrutura consoante-vogal-consoante } \\
\text { Limitação de sillabas }\end{array}$ \\
\hline Preston et al. ${ }^{(22)}$ & Dificuldade com palavras multissilábicas \\
\hline
\end{tabular}

Fonte: Elaborado pelas autoras (2020).

No quadro 4 , as alterações estão agrupadas conforme semelhanças e contabilizadas quanto ao aparecimento nos artigos, e a inconsistência em diversos tipos de erros, como exclusões, substituições, inversões e distorções de sílabas, vogais e consoantes foi a alteração mais constante (13 artigos). Foi seguida de simplificação de palavras, dificuldade com palavras multissilábicas ou aumento na proporção de erros a partir de expressões mais extensas e complexas (9 artigos), variações ou inadequações articulatórias (6 artigos), dificuldade em combinar fonemas ou na estrutura consoante-vogal-consoante/ sequenciamento (5 artigos), reiteração de 
oposições consonantais com sons parecidos, alteração da pauta acentual e da entonação (1 associados também com vogais/ dissílabas com artigo), e pausas aumentadas entre palavras, sílabas duplicadas, com mesma consoante e, sílabas e fonemas (1 artigo). principalmente, palavras polissilábicas (2 artigos), Quadro 4. Aparecimento das alterações nos artigos.

\begin{tabular}{|c|c|}
\hline Principais alterações de linguagem oral & N \\
\hline Variações ou inadequações articulatórias & 6 \\
\hline $\begin{array}{c}\text { Inconsistência em diversos tipos de erros (exclusões, } \\
\text { substituições, inversões e distorções de sílabas, vogais ou } \\
\text { consoantes) }\end{array}$ & 13 \\
\hline $\begin{array}{c}\text { Dificuldade em combinar sons/ Mau sequenciamento/ Dificuldades } \\
\text { na estrutura consoante-vogal-consoante }\end{array}$ & 5 \\
\hline $\begin{array}{c}\text { Pausas aumentadas entre palavras, sílabas e sons } \\
\text { Simplificação de palavras/ Dificuldade com palavras } \\
\text { expressões mais extensas e complexas }\end{array}$ & $\mathbf{1}$ \\
\hline $\begin{array}{c}\text { Reiteração de oposições consonantais com sons } \\
\text { parecidos, associados também com vogais/ Dissílabas } \\
\text { com sílabas duplicadas, com mesma consoante e, } \\
\text { principalmente, palavras polissilábicas }\end{array}$ & 2 \\
\hline \begin{tabular}{c} 
Alteração da pauta acentual e da entonação \\
\hline
\end{tabular} & 1 \\
\hline
\end{tabular}

Fonte: Elaborado pelas autoras (2020).

\section{DISCUSSÃO}

O enfoque da apraxia de fala relacionada ao público infanto-juvenil e também com causas idiopáticas é um assunto controverso, pois há uma redução de trabalhos na literatura focados nesta temática. Foram observadas dificuldades para a seleção de artigos voltados para a área, pois a maioria das pesquisas de campo sobre apraxia não distinguem indivíduos apráxicos idiopáticos dos que possuem a apraxia apenas como sinal de uma patologia base, assim como não distinguem crianças e adultos.

O estudo de Souza e Payão ${ }^{(5)}$ foi o único encontrado cujo método compartilhava semelhanças, e estes autores identificaram durante o levantamento de dados em seus artigos selecionados, determinadas alterações em comum, como fala ininteligível, inconsistência nos erros, sequenciamento de sílabas prejudicado, erros em padrões fonéticos, repetição de palavras ilógicas, variação de acentuação restrita e cadência de fala comprometida, resultados compatíveis em grande parte com os desta pesquisa.

A inconsistência nos erros, alteração que apareceu no presente estudo em quase todos os artigos analisados e uma das mais proeminentes da apraxia, é detalhada por Almeida-Verdu et al. $^{(9)}$, pois o autor esclarece que o paciente pode produzir corretamente uma palavra em determinada situação e, com erros, em outra.

Terband et al. ${ }^{(23)}$ define de forma mais pontual que todas as alterações presentes na linguagem oral de sujeitos com apraxia derivam somente de uma alteração primária: articulação, pois a programação voluntária dos movimentos 
orofaciais para emissão de fonemas está REFERÊNCIAS

prejudicada.

\section{CONCLUSÃO}

Esta pesquisa verificou o cenário nacional e internacional através de publicações atuais sobre as principais alterações identificadas na linguagem oral de pacientes infantis e jovens apráxicos idiopáticos. A maior parte dos sujeitos avaliados no artigos selecionados apresentaram inconsistência em diversos erros, como exclusões, substituições, inversões e distorções de sílabas, vogais e consoantes, simplificação de palavras, dificuldade com palavras multissilábicas ou aumento na proporção de erros a partir de expressões mais extensas e complexas, e variações ou inadequações articulatórias.

A literatura observada foi pequena em termos quantitativos, o que revela a necessidade de mais pesquisas não-generalistas, ou seja, que busquem mais dados científicos voltados para as diferentes origens do distúrbio e públicos também diversos. Vale lembrar que cada distúrbio possui características singulares, e portanto, precisam ser estudados de acordo com suas particularidades, para que possa haver maior precisão em diagnósticos e processos terapêuticos precoces com direcionamento.

1. Silva CAPPG. Transtornos fonéticofonológicos na Síndrome de Down e implicações na lectoescrita. SCRIPTA. 2010; 14(26): 57-70.

2. American Speech-Language-Hearing Association. Childhood Apraxia of Speech [Acesso em 22 mar 2020]. Disponível em: https://www.asha.org/ PRPSpecific'Topic.aspx?folderid=8589935338\&section =Causes.

3. Shriberg LD, Fourakis M, Karlsson H, Lohmeier HL, Mcsweeny JL. Extensions to the Speech Disorders Classification System (SDCS). Clin Linguist Phon. 2010; 24(10): 795-824.

4. Lewis BA, Freebairn LA, Hansen AJ, Iyengar SK, Taylor HG. Schoolage follow-up of children with childhood apraxia of speech. Lang Speech Hear Serv Sch. 2004; 35(2): 122-40.

5. Souza TNU, Payão LMC. Apraxia da fala adquirida e desenvolvimental: semelhanças e diferenças. Rev. Soc. Bras. Fonoaudiol. 2008; 13(2): 193-202.

6. Iuzzini J, Forrest K. Evaluation of a combined treatment approach for childhood apraxia of speech. Clin Linguist Phon. 2010; 24(4-5): 335-45.

7. Rechia IC, Souza APR, Mezzommo CL. Processos de Apagamento na fala de sujeitos com Dispraxia Verbal, Rev. CEFAC. 2010; 12(3): 421-426.

8. Rechia IC, Souza APR, Mezzommo CL, Moro MP. Processos de substituição e variabilidade articulatória na fala de sujeitos com dispraxia verbal. Rev. Soc. Bras. Fonoaudiol. 2009; 14(4): 547-552.

9. Almeida-Verdu ACM, Giacheti CM, Lucchesi FDM. Apraxia e produção da fala: efeitos do fortalecimento de relações verbais. Rev. CEFAC. 2015; 17(3): 974-983.

10. Rechia IC, Souza APR. Dialogia e função materna em casos de limitações práxicas verbais. Rev. Psicol. Estud. 2010; 15(2): 315-23.

11. Catrini M, Lier-Devitto MF. Apraxia de fala e atraso de linguagem: a complexidade do diagnóstico e tratamento em quadros sintomáticos de crianças. CoDAS. 2019; 31(5): 1-6.

12. Grigos MI, Moss A, Lu Y. Oral Articulatory Control in Childhood Apraxia of Speech. J Speech Lang Hear Res. 2015; 58(4): 1103-1118.

13. Grigos MI, Kolenda N. The relationship between articulatory control and improved phonemic accuracy in childhood apraxia of speech: A longitudinal case study. Clin Linguist Phon. 2010; 24(1): 17-40.

14. Fedorenko E, Morgan A, Murray E. A highly penetrant form of childhood apraxia of speech due to deletion of 16p11.2. Eur J Hum Genet. 2016; 24: 302306.

15. Keske-Soares M, Uberti LB, Gubiani MB, Gubiani MB, Ceron MI, Pagliarin KC. Desempenho de crianças com distúrbios dos sons da fala no instrumento "Avaliação dinâmica das habilidades motoras da fala". CoDAS. 2018; 30 (2).

16. Murray E, Thomas D, Mckechnie J. Comorbid 
morphological disorder apparent in some children aged 4-5 years with childhood apraxia of speech: findings from standardised testing. Clin Linguist Phon. 2019; 33(1-2): 42-59.

17. Shakibayi MI, Zarifian T, Zanjari N. Speech characteristics of childhood apraxia of speech: A survey research. Int J Pediatr Otorhinolaryngol. 2019; 126.

18. Merwe A, Steyn M. Model-Driven Treatment of Childhood Apraxia of Speech: Positive Effects of the Speech Motor Learning Approach. Am J Speech Lang Pathol. 2018; 27(1): 37-51.

19. Malmenholt A, Lohmander A, Mcallister A. Childhood apraxia of speech: A survey of praxis and typical speech characteristics. Logoped Phoniatr Vocol. 2017; 42(2): 84-92.

20. Case J, Grigos MI. Articulatory Control in Childhood Apraxia of Speech in a Novel Word-Learning Task. J Speech Lang Hear Res. 2016; 59(6): 1253-1268.

21. Overby M, Caspari SS. Volubility, consonant, and syllable characteristics in infants and toddlers later diagnosed with childhood apraxia of speech: A pilot study. J Commun Disord. 2015; 55: 44-62.

22. Preston JL, Molfese PJ, Gumkowski N, Sorcinelli A, Harwood V, Irwin JR, Landi N. Neurophysiology of speech differences in childhood apraxia of speech. Developmental Neuropsychology. 2014; 39(5): 385-403. 23. Terband H, Rodd J, Maas E. Testing hypotheses about the underlying deficit of apraxia of speech through computational neural modelling with the DIVA model. I J Speech Lang Pathol. 2019.

OBSERVAÇÃO: Os autores declaram não existir conflitos de interesse de qualquer natureza. 